\title{
Accurate and Conforming Mixed Discretization of the MFIE
}

\author{
K. Cools, F. P. Andriulli, D. De Zutter, and E. Michielssen
}

\begin{abstract}
In this contribution, a novel discretization scheme for the magnetic field integral equation is presented. The new scheme is designated "mixed" because it uses Rao-Wilton-Glisson functions to expand the current density and Buffa-Christiansen functions to test the magnetic field radiated by the candidate solution. The convergent nature of the proposed mixed MFIE is theoretically proven and numerical results showing that the proposed method yields more accurate results than the classical one are presented.
\end{abstract}

\section{INTRODUCTION}

Scattering of time-harmonic electromagnetic waves by perfect electrically conducting (PEC) surfaces can be modelled by many boundary integral equations, the electric and magnetic field integral equations (EFIE and MFIE) being the most prominent ones [1]. These equations typically are discretized by expanding the current density in terms of Rao-WiltonGlisson (RWG) functions defined on a triangular mesh that approximates the scatterer's surface and testing the equations using the same RWG functions [2].

The EFIE often yields highly accurate results, is applicable to both open and closed structures, and extendable to impedance sheets. Regrettably, the linear systems that result from its discretization have unbounded condition numbers in the dense mesh regime, leading to prohibitive solution times. This problem can be solved by Calderón preconditioning (see [3] and references therein), i.e. by exploiting the EFIE operator's self-regularizing property. A Calderón preconditioned EFIE system basically represents a discretized second kind integral equation and thus is amenable to efficient iterative solution. Alternatively, a multi-resolution basis can be used for the finite element spaces of expansion and testing functions (see [4] and references therein). The singular value spectrum of the resulting linear system once again is bounded from above and below, thus facilitating its efficient iterative solution.

The MFIE, in contrast, upon discretization yields wellconditioned systems without further manipulations. Unfortunately, the MFIE's solution is less accurate than that of the EFIE, with a numerical error that can be up to several orders of magnitude larger than that of the EFIE. In the past, strategies to reduce the error in the solution of classically discretized MFIEs have been proposed [5], [6], [7].

In this work, starting from the function space mapping properties of the MFIE operator, a novel discretization scheme is proposed. The convergence properties of the new scheme are

This work was supported in part by AFOSR MURI Grant F014432-051936 aimed at modeling installed antennas and their feeds, by NSF Grant DMS 0713771, and in part by DARPA-DSO/AFOSR Grant F015123-052587 aimed at constructing compressed scattering matrices and direct solvers. rigorously investigated and numerically verified. The accuracy improvements realized by the new discretization scheme over the classical one are quantified. Very preliminary results, without proof, were included in conference proceedings [8].

\section{EQUATIONS AND DISCRETIZATION}

Consider a closed PEC scatterer with surface $\Gamma$ and exterior normal $\hat{\boldsymbol{n}}$, embedded in a background medium with permittivity $\epsilon$ and permeability $\mu$. The scatterer is illuminated by an incident electromagnetic field $\left(\boldsymbol{e}^{i}, \boldsymbol{h}^{i}\right)$. Enforcing the boundary conditions for the tangential traces of the electric and magnetic fields on $\Gamma$ leads to the following EFIE and MFIE for the induced current density $j$ :

$$
\begin{aligned}
& -\hat{\boldsymbol{n}} \times \hat{\boldsymbol{n}} \times \boldsymbol{e}^{i}(\boldsymbol{r})=\eta S[\boldsymbol{j}](\boldsymbol{r}) \\
:= & \hat{\boldsymbol{n}} \times \hat{\boldsymbol{n}} \times \frac{1}{4 \pi \epsilon} \frac{1}{j \omega} p \cdot v \cdot \int_{\Gamma} \nabla \frac{e^{-j k R}}{R} \nabla^{\prime} \cdot \boldsymbol{j}\left(\boldsymbol{r}^{\prime}\right) d S^{\prime} \\
& -\hat{\boldsymbol{n}} \times \hat{\boldsymbol{n}} \times \frac{\mu}{4 \pi} j \omega \int_{\Gamma} \frac{e^{-j k R}}{R} \boldsymbol{j}\left(\boldsymbol{r}^{\prime}\right) d S^{\prime} . \\
& \hat{\boldsymbol{n}} \times \boldsymbol{h}^{i}(\boldsymbol{r})=\left\{\frac{1}{2}-K\right\}[\boldsymbol{j}](\boldsymbol{r}) \\
:= & \frac{1}{2} \boldsymbol{j}(\boldsymbol{r})-\hat{\boldsymbol{n}} \times \frac{1}{4 \pi} \int_{\Gamma} \frac{e^{-j k R}}{R} \times \boldsymbol{j}\left(\boldsymbol{r}^{\prime}\right) d S^{\prime},
\end{aligned}
$$

To solve this EFIE and MFIE via a Galerkin boundary element method, the surface $\Gamma$ is discretized by a mesh of planar triangles with mesh parameter (i.e. largest triangle diameter) $h$. The current $j$ is approximated as

$$
\boldsymbol{j}(\boldsymbol{r}) \approx \sum_{i=n}^{N} I_{n} \boldsymbol{f}_{n}(\boldsymbol{r})
$$

where the functions $\boldsymbol{f}_{n}$ are RWG basis functions defined on the $N$ interior edges of the mesh [2]. Equation (3) is substituted in (1) and (2) and the Galerkin procedure is completed by testing the two equations with $N$ appropriately chosen basis functions.

The choice of the testing basis functions should follow the following rationale: if the operator to be discretized with a Galerkin procedure maps function space $X$ to function space $Y$, then the testing functions should be in function space $Y^{*}$, viz. the dual of $Y$ [9].

In classical implementations both the EFIE and the MFIE are tested by the same RWG basis functions used to expand the current. It is well-known that the RWG basis functions belong to function space $H^{-1 / 2}(\operatorname{div} ; \Gamma)$, viz. the space of functions 
$j$ for which

$\iint_{\Gamma \times \Gamma} \frac{\boldsymbol{j}(\boldsymbol{r}) \cdot \overline{\boldsymbol{j}\left(\boldsymbol{r}^{\prime}\right)}}{R} d S^{\prime} d S+\iint_{\Gamma \times \Gamma} \frac{\operatorname{div} \boldsymbol{j}(\boldsymbol{r}) \overline{\operatorname{div}^{\prime} \boldsymbol{j}\left(\boldsymbol{r}^{\prime}\right)}}{R} d S^{\prime} d S$

is finite. Here, the bar indicates complex conjugation. It can be shown that the dual of the space $H^{-1 / 2}(\operatorname{div} ; \Gamma)$ is $H^{-1 / 2}(\operatorname{curl} ; \Gamma)$ [10], viz. the space of functions $j$ for which $\hat{\boldsymbol{n}} \times \boldsymbol{j}$ belongs to $H^{-1 / 2}(\operatorname{div} ; \Gamma)$. Likewise, the dual of $H^{-1 / 2}(\operatorname{curl} ; \Gamma)$ is $H^{-1 / 2}(\operatorname{div} ; \Gamma)$ [10]. Another well-known result from operator theory [10] is that the EFIE operator $S$ maps $H^{-1 / 2}(\operatorname{div} ; \Gamma)$ into $H^{-1 / 2}(\operatorname{curl} ; \Gamma)$, so that the testing should be done using functions in $H^{-1 / 2}(\operatorname{div} ; \Gamma)$, in accordance with the classical choice of RWGs as testing functions for the EFIE. For the MFIE, however, the situation is different: the MFIE operator $\frac{1}{2}-K$ maps $H^{-1 / 2}(\operatorname{div} ; \Gamma)$ into itself (as is suggested by the presence of the identity) and a conformal testing requires that the testing functions are in $H^{-1 / 2}(\operatorname{curl} ; \Gamma)$. The RWG functions, commonly used to test the MFIE, do not reside in $H^{-1 / 2}(\operatorname{curl} ; \Gamma)$, but in $H^{-1 / 2}(\operatorname{div} ; \Gamma)$. In this paper we present a method for testing the MFIE that, in contrast to the commonly used procedure, uses testing functions in $H^{-1 / 2}(\operatorname{curl} ; \Gamma)$.

As stated above, in the classical discretization scheme, RWG functions $\boldsymbol{f}_{n}$ (that reside in $H^{-1 / 2}(\operatorname{div} ; \Gamma)$ ) are used to test the MFIE, the rationale being that this choice renders the matrix discretizing the identity operator well-conditioned. Since the testing functions should instead have been selected in $H^{-1 / 2}$ (curl; $\Gamma$ ), it may appear befitting to use "rotated" RWG functions $\hat{\boldsymbol{n}} \times \boldsymbol{f}_{n}$ (that reside in $H^{-1 / 2}(\operatorname{curl} ; \Gamma)$ ) as testing functions. However, this choice renders the matrix discretizing the identity operator singular, resulting in an ill-conditioned discretization of the MFIE. To achieve a conforming and wellconditioned discretization of the MFIE, it suffices to find a set of testing functions that resides in $H^{-1 / 2}(\operatorname{curl} ; \Gamma)$ and results in a well-conditioned discretization of the identity operator when used in combination with RWG expansion functions. The set of "rotated" Buffa-Christiansen (BC) functions $\hat{\boldsymbol{n}} \times \boldsymbol{g}_{n}$, defined on the barycentric refinement of the original barycentric mesh, constitutes such a set. For brevity the reader is referred to [11] for the definition and properties of these functions.

The above described discretization scheme of the MFIE gives rise to the following boundary element formulation. The current density is approximated as

$$
\boldsymbol{j}_{h}(\boldsymbol{r})=\sum_{i=n}^{N} I_{n} \boldsymbol{f}_{n}(\boldsymbol{r})
$$

such that

$$
\left(\frac{1}{2} \mathbf{G}+\mathbf{K}\right) \cdot \mathrm{I}=\mathrm{H}
$$

where $(\mathrm{I})_{n}=I_{n}$,

$$
\begin{gathered}
(\mathbf{G})_{m, n}=\int_{\Gamma}\left(\hat{\boldsymbol{n}} \times \boldsymbol{g}_{m}(\boldsymbol{r})\right) \cdot \boldsymbol{f}_{n}(\boldsymbol{r}) d S \\
(\mathbf{K})_{m, n}=-\frac{1}{4 \pi} \iint_{\Gamma \times \Gamma} \boldsymbol{g}_{m}(\boldsymbol{r}) \cdot\left(\nabla \frac{e^{-j k R}}{R} \times \boldsymbol{f}_{n}\left(\boldsymbol{r}^{\prime}\right)\right) d S^{\prime} d S,
\end{gathered}
$$

$$
(\mathrm{H})_{m}=\int_{\Gamma} \boldsymbol{g}_{m}(\boldsymbol{r}) \cdot \boldsymbol{h}^{i}(\boldsymbol{r}) d S .
$$

In [11], the well-conditioned nature of the discretized identity operator $\mathbf{G}$ was proven. Equation (8) reveals another advantage of the proposed discretization scheme used. Since the cross product with the normal vector, present in classical MFIE formulations, has disappeared, it is easy to transfer the gradient operator from the Green function onto the testing function using the methods described in [12]. By doing this, the logarithmic singularity appearing in the magnetic field radiated by an RWG function can be avoided. It is this singularity that, when using classical schemes, prohibits the accurate evaluation of the MFIE system matrix' elements.

The proposed scheme is convergent. To prove this, assume that $\boldsymbol{j}$ is the exact solution of the MFIE, that $\boldsymbol{j}_{h}$ is the solution of the discretized equation (6), that $j^{\prime}$ is a generic function in the space of RWG functions, and that $\boldsymbol{k}^{\prime}$ is a generic function in the space of $\mathrm{BC}$ functions. In what follows the spaces of RWG and $\mathrm{BC}$ functions are denoted as $R W G$ and $B C$, respectively. Also, let $\sigma_{\min }$ denote the minimum singular value of $\mathbf{K}-\frac{1}{2} \mathbf{G}$. Then, using the min-max characterization of singular values [13], it can be seen that the following inequality holds

$$
\left\|\boldsymbol{j}^{\prime}-\boldsymbol{j}_{h}\right\| \lesssim \frac{1}{\sigma_{\min }} \sup _{\boldsymbol{k}^{\prime} \in B C} \frac{\left(\boldsymbol{k}^{\prime},\left\{K-\frac{1}{2}\right\}\left(\boldsymbol{j}^{\prime}-\boldsymbol{j}_{h}\right)\right)}{\left\|\boldsymbol{k}^{\prime}\right\|} .
$$

Using this inequality, the discretization error, i.e. the distance between the solution $j_{h}$ of the discretized MFIE and the exact solution $j$ of the MFIE can be bounded as

$$
\left\|\boldsymbol{j}-\boldsymbol{j}_{h}\right\| \leq\left\|\boldsymbol{j}-\boldsymbol{j}^{\prime}\right\|+\left\|\boldsymbol{j}^{\prime}-\boldsymbol{j}_{h}\right\|
$$

with

$$
\begin{aligned}
\left\|\boldsymbol{j}^{\prime}-\boldsymbol{j}_{h}\right\| & \lesssim \frac{1}{\sigma_{\min }} \sup _{\boldsymbol{k}^{\prime} \in B C} \frac{\left(\boldsymbol{k}^{\prime},\left\{K-\frac{1}{2}\right\}\left(\boldsymbol{j}^{\prime}-\boldsymbol{j}_{h}\right)\right)}{\left\|\boldsymbol{k}^{\prime}\right\|} \\
& =\frac{1}{\sigma_{\min }} \sup _{\boldsymbol{k}^{\prime} \in B C} \frac{\left(\boldsymbol{k}^{\prime},\left\{K-\frac{1}{2}\right\}\left(\boldsymbol{j}^{\prime}-\boldsymbol{j}\right)\right)}{\left\|\boldsymbol{k}^{\prime}\right\|} \\
& \lesssim \frac{1}{\sigma_{\min }} \sup _{\boldsymbol{k}^{\prime} \in B C} \frac{\left\|\boldsymbol{k}^{\prime}\right\|\left\|\left\{K-\frac{1}{2}\right\}\right\|\left\|\left(\boldsymbol{j}^{\prime}-\boldsymbol{j}\right)\right\|}{\left\|\boldsymbol{k}^{\prime}\right\|} \\
& \lesssim \frac{1}{\sigma_{\min }}\left\|\boldsymbol{j}^{\prime}-\boldsymbol{j}\right\| \quad \forall \boldsymbol{j}^{\prime} \in R W G .
\end{aligned}
$$

In deriving (12) we used the continuity of the operator $\left\{K-\frac{1}{2}\right\}$ and the fact that

$$
\left(\boldsymbol{k}^{\prime},\left\{K-\frac{1}{2}\right\} \boldsymbol{j}_{h}\right)=\left(\boldsymbol{k}^{\prime},\left\{K-\frac{1}{2}\right\} \boldsymbol{j}\right) \quad \forall \boldsymbol{k}^{\prime} \in B C .
$$

The latter follows immediately from the construction in (6) of the discrete solution $j_{h}$. It can thus be concluded that

$$
\left\|\boldsymbol{j}-\boldsymbol{j}_{h}\right\| \lesssim\left(1+\frac{1}{\sigma_{\min }}\right)\left\|\boldsymbol{j}-\boldsymbol{j}^{\prime}\right\| \quad \forall \boldsymbol{j}^{\prime} \in R W G .
$$

The best approximation of $\boldsymbol{j}$ in the RWG space involves errors in the order of $h$ [14], i.e.

$$
\inf _{\boldsymbol{j}^{\prime} \in R W G}\left\|\boldsymbol{j}^{\prime}-\boldsymbol{j}\right\| \lesssim h\|\boldsymbol{j}\| .
$$


By combining (14) with (15), we obtain

$$
\left\|\boldsymbol{j}-\boldsymbol{j}_{h}\right\| \lesssim h\left(1+\frac{1}{\sigma_{\min }}\right)\|\boldsymbol{j}\|
$$

This means that if $\sigma_{\min }$ is bounded from below, uniformly with respect to the mesh parameter $h$, the discretized solution $\boldsymbol{j}_{h}$ converges to the exact solution $\boldsymbol{j}$ as $h \rightarrow 0$. Since the MFIE is a second kind equation [15] and the matrix $\mathbf{G}$ is well-conditioned regardless of the mesh parameter $h$ [11], the above two conditions are satisfied provided that the scatterer does not support resonances at the simulation frequency.

\section{NUMERICAL RESULTS}

The proposed scheme was applied to the analysis of scattering from a sphere of radius $1 \mathrm{~m}$, modelled in terms of increasingly dense meshes. The coarsest mesh involves $N=$ 147 degrees of freedom and has mesh parameter $h=0.86 \mathrm{~m}$, while the finest mesh has $N=2430$ and $h=0.22 \mathrm{~m}$. The sphere is illuminated by the plane wave

$$
\boldsymbol{e}^{i}(\boldsymbol{r})=\hat{\boldsymbol{x}} e^{-j \frac{2 \pi}{\lambda} z}
$$

with $\lambda=2$ meters. The scattering problem was solved using the EFIE, the classic (non-conformingly discretized) MFIE, and the mixed (conformingly discretized) MFIE. The relative $L^{2}(S)$ error of the radar cross section relative to the Mie series solution shows that the mixed MFIE yields results comparable in accuracy to the EFIE, and smaller than that of the classic MFIE (Fig. 1(a)). Next, a mesh with fixed mesh parameter $h$ is chosen, and the scattering problem again solved using the EFIE, the classical MFIE, and the mixed MFIE for a set of frequencies in the band from 15 to $480 \mathrm{MHz}$. For every frequency considered, the mixed MFIE yields results comparable in accuracy to the EFIE, but more accurate than those of the classical MFIE.

Finally, the above exercise was repeated for a cube with side length of $1 \mathrm{~m}$. The coarsest mesh has $N=210$ and $h=0.28 \mathrm{~m}$, while the finest mesh has $N=4896$ and $h=0.04 \mathrm{~m}$. The cube is illuminated by the field in (17). Since no analytical solution is available for this problem, the $L^{2}(S)$ errors were computed w.r.t the EFIE's solution for the finest mesh. It is again clear that the mixed MFIE outperforms the classic one, this both when the mesh is refined (Fig. 2(a)) and, for a fixed mesh, when the frequency is varied (Fig. 2(b)).

\section{CONCLUSIONS}

A novel "mixed" discretization scheme for the MFIE has been presented. The new scheme uses Rao-Wilton-Glisson functions to expand the current density and Buffa-Christiansen functions as testing functions. The convergence properties of the proposed MFIE have been rigorously proven and numerical results have been presented showing that the proposed mixed MFIE yields more accurate solutions than the classical one. Future investigations will concentrate on the application of the mixed discretization strategy, proposed here, to the discretization of the combined field integral equation for PEC scatterers, the Muller and PMCHWT integral equations for penetrable objects, and integral equations for scatterers characterized by an impedance boundary condition.

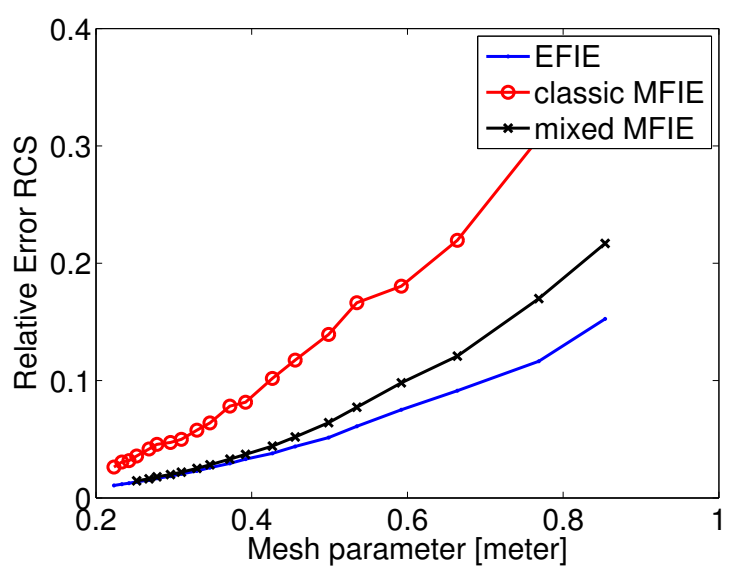

(a)

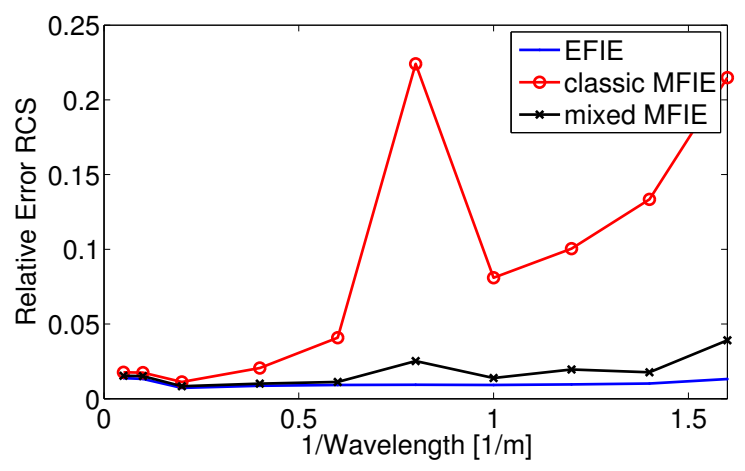

(b)

Fig. 1. The relative $L^{2}(S)$-error of the RCS for a sphere as a function of the mesh parameter (a) and as a function of the simulation frequency (b)

\section{REFERENCES}

[1] J. V. Bladel, Electromagnetic Fields. IEEE Press, 2007.

[2] S. M. Rao, D. Wilton, and A. Glisson, "Electromagnetic scattering by surfaces of arbitrary shape," IEEE Trans. Antennas Propagation, vol. 30, pp. $408-418,1982$.

[3] F. P. Andriulli, K. Cools, H. Bagci, F. Olyslager, A. Buffa, S. Christiansen, and E. Michielssen, "A Multiplicative Calderon Preconditioner for the Electric Field Integral Equation," IEEE Trans. Antennas Propagation, vol. 56, no. 8, pp. 2398-2412, 2008.

[4] F. P. Andriulli, A. Tabacco, and G. Vecchi, "Solving the EFIE at Low Frequencies With a Conditioning That Grows Only Logarithmically With the Number of Unknowns," Antennas and Propagation, IEEE Transactions on, vol. 58, pp. 1614 -1624, may. 2010.

[5] Y. Zhang, T. Cui, W. Chew, and J.-S. Zhao, "Magnetic field integral equation at very low frequencies," IEEE Transactions on Antennas and Propgation, vol. 51, pp. 1864-1871, August 2003.

[6] O. Ergul and L. Gurel, "Improved testing of the magnetic field integral equation," IEEE Microwave and Wireless Communication Letters, vol. 15, pp. 615-617, October 2005.

[7] E. Ubeda and J. Rius, "Novel monopolar mfie mom-discretization for the scattering analysis of small objects," IEEE Transactions on Antennas and Propgation, vol. 54, pp. 50-57, January 2006.

[8] K. Cools, F. Andriulli, F. Olyslager, and E. Michielssen, "Improving the mfie's accuracy by using a mixed discretization," in Antennas and Propagation Society International Symposium, pp. 1-4, June 2009.

[9] W. Rudin, Functional Analysis. McGraw-Hill, 2003.

[10] M. Cessenat, Mathematical Methods in Electromagnetism: Linear Theory and Applications. World Scientific Publishing Company, 1996.

[11] A. Buffa and S. H. Christiansen, "A dual finite element complex on the barycentric refinement," Tech. Report PV-18 IMATI-CNR, 2005.

[12] P. Ylä-Oijala and M. Taskinen, "Calculation of CFIE impedance matrix elements with RWG and n x RWG functions," IEEE Transactions on Antennas and Propagation, vol. 51, pp. 1837-1846, August 2003. 


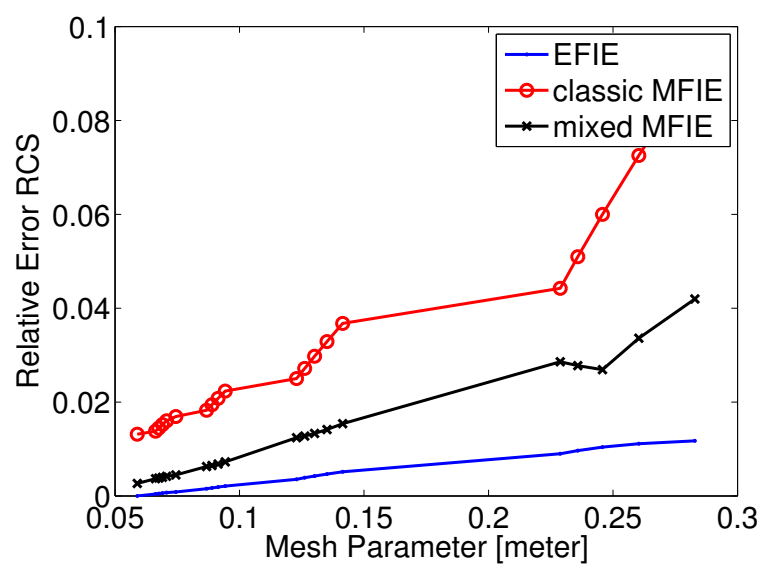

(a)

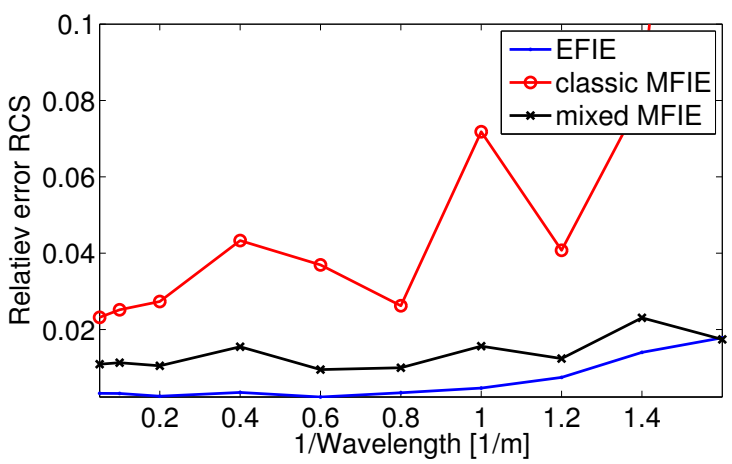

(b)

Fig. 2. The relative $L^{2}(S)$-error of the RCS for a cube as a function of the mesh parameter (a) and as a function of the simulation frequency (b)

[13] G. H. Golub and C. F. V. Loan, Matrix Computations. Baltimore, MD, USA: Johns Hopkins Press, second ed., 1989.

[14] A. Quarteroni and A. Valli, Numerical Approximation of Partial Differential Equations. Springer, 1997.

[15] I. Goldberg, S. Kaashoek, and A. Marinus, Basic Classes of Linear Operators. Birkhauser, 2003. 